\title{
Vibration analysis by speckle techniques in real time
}

\author{
H. J. Tiziani and J. Klenk
}

\begin{abstract}
Vibration analysis in quasi-real time is studied by recording the time-averaged speckle pattern in a bismuth silicon oxide crystal $\left(\mathrm{Bi}_{12} \mathrm{SiO}_{20}\right)$. When the crystal is illuminated with a spherical wave, the spatially structured information from the time-averaged speckle pattern leads to fringes in the Fraunhofer plane. No storage device is required when the appropriate wavelength is chosen for writing and reading out the information. Experimental results will be presented together with a comparison of measurements obtained with a Doppler shift vibrometer.
\end{abstract}

\section{Introduction}

Holographic interferometry and speckle applications for deformation, displacement, and vibration analysis are becoming important methods. They will be even more attractive to the engineer when applied in real time. Some crystals such as bismuth silicon oxide, $\mathrm{Bi}_{12} \mathrm{SiO}_{20}$ (BSO), have electrical and electrooptical properties making them attractive for dynamic holography and speckle applications. The basic principle for the application of the crystal to holographic storage and double-exposure interferometry of transparent or mirrorlike structures has been reported. ${ }^{1-3}$ Real-time speckle applications have been reported in Ref. 4 . Real-time speckle recording using a liquid crystal light valve as the recording material has been reported in Ref. 5.

For real-time speckle applications the BSO crystal is usually biased with a transverse electric field $E_{0}$ in the 110 crystallographic direction. When the crystal is illuminated with spatially structured illumination as a result of a speckle pattern in the $\overline{1} 10$ direction, a space charge field is built up. The physical mechanisms for speckle pattern recording and erasure in BSO are drift and trapping of photoelectrons under illumination by a random light pattern field in the transverse electrooptic configuration. The photoinduced space charge field changes the refractive index of the crystal via the linear electrooptic effect, leading to a refractive-index

The authors are with Universität Stuttgart, Institut für Technische Optik, Pfaffenwaldring 9, 7000 Stuttgart 80, Federal Republic of Germany.

Received 13 November 1980.

0003-6935/81/081467-04\$00.50/0.

(C) 1981 Optical Society of America. variation in the crystal volume. Flooding with uniform illumination leads to the erasure of the stored information by space charge relaxation. Consequently, reading out with the recording wavelength is destructive.

In the particular speckle application, the speckle recording wavelength $\lambda_{1}$ is chosen to be different from the wavelength used to display the fringes. The crystal absorption coefficients are $\alpha_{1}=2 \mathrm{~cm}^{-1}$ for $\lambda_{1}$ and $\alpha_{2}=$ $0.28 \mathrm{~cm}^{-1}$ for $\lambda_{2}$; hence reading out Young's fringes with $\lambda_{2}$ does not require an image storage device.

\section{In-Plane Vibration Analysis by Time-Averaged Recording of the Oscillating Speckle Patterns in a BSO Crystal}

A harmonically oscillating object produces a timevarying speckle pattern. It was found appropriate to record the oscillating speckle pattern in the BSO as a time-average exposure. To simplify the following analysis the Bragg volume effect as well as the birefringence and crystal activity are neglected. The intensity of the Speckle pattern in the image plane can be written according to Fig. 1 using 1-D notation only ${ }^{6}$ :

$$
I^{\prime}\left(u^{\prime}\right)=\sum_{q} \sum_{p} \psi\left(x_{p}^{\prime}\right) \psi^{*}\left(x_{q}^{\prime}\right) \exp \left[i 2 \pi u^{\prime}\left(x_{p}^{\prime}-x_{q}^{\prime}\right)\right],
$$

where $\quad u^{\prime}=$ reduced coordinates, $u^{\prime}=\left[\left(n^{\prime} \sin \alpha^{\prime}\right) /\right.$ $\lambda] \xi^{\prime}$

$\xi^{\prime}=$ rectangular coordinates in the image plane;

$\alpha^{\prime}=$ aperture angle in image space;

$x=X / h, x^{\prime}=X^{\prime} / h^{\prime}=$ reduced coordinates in the entrance and exit pupil; $x \approx x^{\prime}$

$h, h^{\prime}=$ entrance and exit pupil heights, and

$x_{p}, x_{q}, x_{p}^{\prime}, x_{q}^{\prime}=$ reduced coordinates in the entrance and exit pupil plane.

The coordinates in the object and entrance pupil follow. 
$\psi\left(x_{p}\right), \psi^{*}\left(x_{q}\right)$ describe the optically rough surface and represent the angular components of the light scattered by it.

In Ref. 4 the double-exposure speckle patterns are recorded in BSO crystals for deformation displacement and tilt analysis. In this short theory of vibration analysis, the time-averaged recording in the crystal is obtained by considering an ideal optical system and a thin crystal:

$$
\begin{aligned}
\left\langle I\left(u^{\prime}, \rho^{\prime}\right)\right\rangle= & C_{0} \frac{1}{T} \int_{-T / 2}^{T / 2} \sum_{q} \sum_{p} \psi\left(x_{p}\right) \psi^{*}\left(x_{q}\right) \\
& \times \exp \left[i 2 \pi\left(x_{p}-x_{q}\right)\left(u^{\prime}-\rho^{\prime} \cos \omega t\right)\right] d t,
\end{aligned}
$$

where $\rho^{\prime}=M \rho$ is the amplitude of oscillation in the image plane, with $\rho$ in the object and $M$ the lateral magnification. By integration over a large number of cycles we find

$$
\begin{aligned}
\left\langle I\left(u^{\prime}, \rho\right)\right\rangle= & \frac{C_{0}}{T} \sum_{q} \sum_{p} \psi\left(x_{p}^{\prime}\right) \psi^{*}+\left(x_{q}\right) J_{0}\left[2 \pi \rho^{\prime}\left(x_{p}-x_{q}\right)\right] \\
& \times \exp \left[i 2 \pi u^{\prime}\left(x_{p}-x_{q}\right)\right] .
\end{aligned}
$$

The refractive-index variation depends on $\left\langle I\left(u^{\prime}, \rho^{\prime}\right)\right\rangle$.

Illuminating the crystal with a coherent wave of wavelength $\lambda_{2}$ and absorption coefficient $\alpha_{2}$, the wave front emerging from the crystal can be written by considering the earlier assumptions:

$$
A\left(u^{\prime}, \rho^{\prime}\right)=A_{0}\left(u^{\prime}\right) C_{1} \exp \left(-\frac{i 2 \pi}{\lambda_{1}} d \Delta n\right),
$$

where

$$
\begin{aligned}
\Delta n & =\Delta n_{s} \frac{C\left\langle I^{\prime}\left(u^{\prime}, \rho^{\prime}\right)\right\rangle}{\left\langle I\left(u^{\prime}\right)\right\rangle}, \\
\Delta n_{s} & =-\frac{n^{3} r E_{s c}}{2},
\end{aligned}
$$

and $\Delta n_{s}=$ refractive-index modulation at saturation;

$n=$ refractive index $(n \approx 2,5$ for $\lambda=633$ $\mathrm{nm}$ );

$r=$ effective electrooptic coefficient;

$\lambda_{1}=$ recording wavelength;

$\lambda_{2}=$ reading out wavelength;

$d=$ crystal thickness;

$E_{s c}=$ space charge field amplitude derived from Poisson's equation ${ }^{2}$ (For speckles with dimensions $>2 \mu \mathrm{m}$, the space charge field is proportional to the applied ${ }^{4}$ electric field $E_{0 .}$ );

$A_{0}\left(u^{\prime}\right)=$ amplitude of the wave illuminating the crystal; it will be considered as a plane wave of constant amplitude.

$C_{1}$ also takes account of the absorption and reflection loss at the crystal interfaces for the illuminating wavelength $\lambda_{2}$ with $\alpha_{2}=0.28 \mathrm{~cm}^{-1} ; \alpha_{2}$ is very much smaller than $\alpha_{1}$ in order to obtain a reconstruction which is not immediately destructive. The refractive-index variation depends on $\Delta n_{s}$ and the normalized time-average speckle pattern. Since the absorption in the thin crystal and the reflection at the interfaces with antireflection coatings are small, they will be neglected.

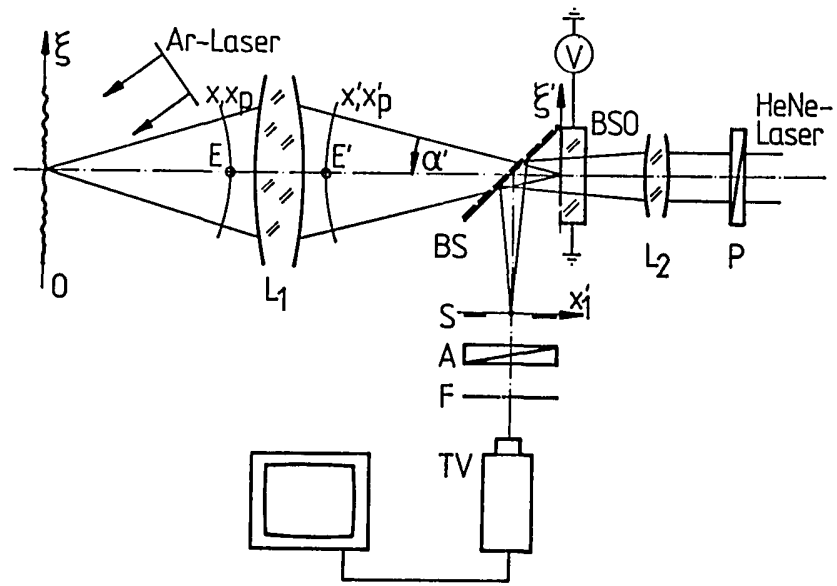

Fig. 1. Experimental arrangement of in-plane deformation and vibration analysis in quasi-real time with an electrooptical BSO crystal.

Furthermore, the photoinduced phase shift is small; $d \Delta n \ll \lambda_{1}$. The amplitude immediately in front of the crystal can, therefore, be written as

$$
\begin{aligned}
A^{\prime}\left(u^{\prime}\right) & =A_{0}^{\prime} C_{1}\left\{1-\frac{i 2 \pi}{\lambda_{1}} d \Delta n\right\} \\
& =A_{0}^{\prime} C_{1}\left\{1-\frac{i 2 \pi}{\lambda_{1}} d \Delta n_{s} \frac{\left\langle I^{\prime}\left(u^{\prime}, \rho^{\prime}\right)\right\rangle}{\left\langle I^{\prime}\left(u^{\prime}\right)\right\rangle}\right\},
\end{aligned}
$$

The Fourier transform $a\left(x_{1}^{\prime}\right)$ of the field amplitude emerging from the crystal is obtained in the focal plane with focal length $f_{2}$. By neglecting constants and a phase factor which will vanish by forming the intensity we can write

$$
\begin{aligned}
a\left(x_{1}^{\prime}\right)= & A_{0}^{\prime} C_{1} \int_{\text {crystal }}\left[1-\frac{i 2 \pi}{\lambda_{1}} d \Delta n_{s} \frac{\left\langle I\left(u^{\prime}, \rho^{\prime}\right)\right\rangle}{\left\langle I\left(u^{\prime}\right)\right\rangle}\right] \\
& \times \exp \left(-\frac{i 2 \pi}{\lambda_{2} f_{2}} u^{\prime} x_{1}^{\prime}\right) d u^{\prime} .
\end{aligned}
$$

By substituting for $I^{\prime}\left(u^{\prime}, \rho^{\prime}\right)$ and writing $x_{r}$ for $\left(x_{p}-x_{q}\right)$ we find

$$
\begin{aligned}
a\left(x_{1}^{\prime}\right)= & A_{0}^{\prime} C_{1}\left[\delta\left(x_{1}^{\prime}\right)-\frac{i 2 \pi}{\lambda_{1}} d \Delta n_{s}\right. \\
& \left.\times \frac{\sum_{q} \psi\left(x_{1}^{\prime}+x_{q}\right) \psi^{*}\left(x_{q}\right)}{\left\langle I\left(u^{\prime}\right)\right\rangle} J_{0}\left(\frac{2 \pi}{\lambda_{2} f_{2}^{\prime}} \rho^{\prime} x_{1}^{\prime}\right)\right],
\end{aligned}
$$

and the intensity in the Fraunhofer plane is

$$
\begin{aligned}
\left|a\left(x_{1}^{\prime}\right)\right|^{2}= & \left|A_{0}^{\prime}\right|^{2} C_{1}^{2}\left[\delta\left(x_{1}^{\prime}\right)+\left(\frac{2 \pi}{\lambda_{1}} d \Delta n_{s}\right)^{2}\right. \\
& \left.\times\left|\frac{\sum_{q} \psi\left(x_{1}^{\prime}+x_{q}\right) \psi^{*}\left(x_{q}\right)}{\left\langle I\left(u^{\prime}\right)\right\rangle}\right|^{2} J_{0}^{2}\left(\frac{2 \pi}{\lambda_{2} f_{2}^{\prime}} \rho^{\prime} x_{1}^{\prime}\right)\right] .
\end{aligned}
$$

The square of $\Sigma_{q} \psi\left(x_{1}+x_{q}\right) \psi^{*}\left(x_{q}\right)$, the autocorrelation 


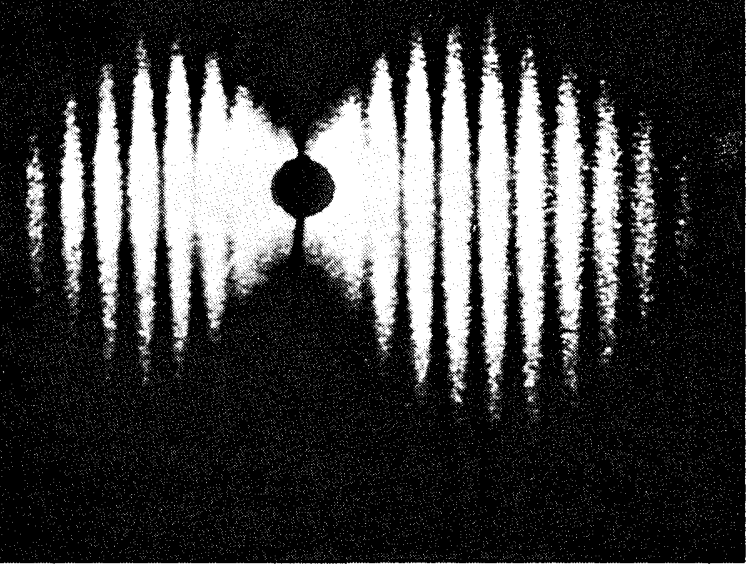

Fig. 2. Real-time fringes of deformation measurement $(\Delta \xi=40-\mu \mathrm{m}$ horizontally).

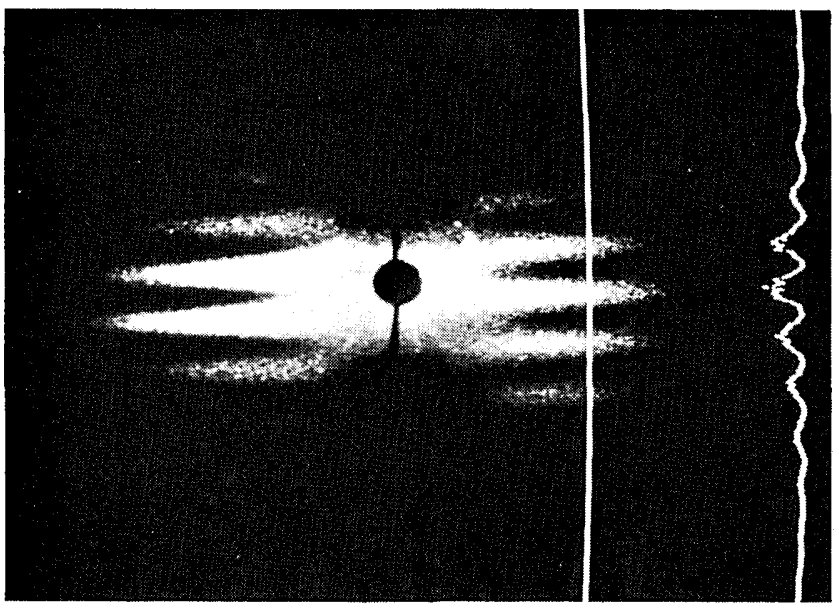

Fig. 3. Real-time fringes of tilt measurement with a TV column analyzer $(\Delta \gamma=20$-sec of arc horizontally).

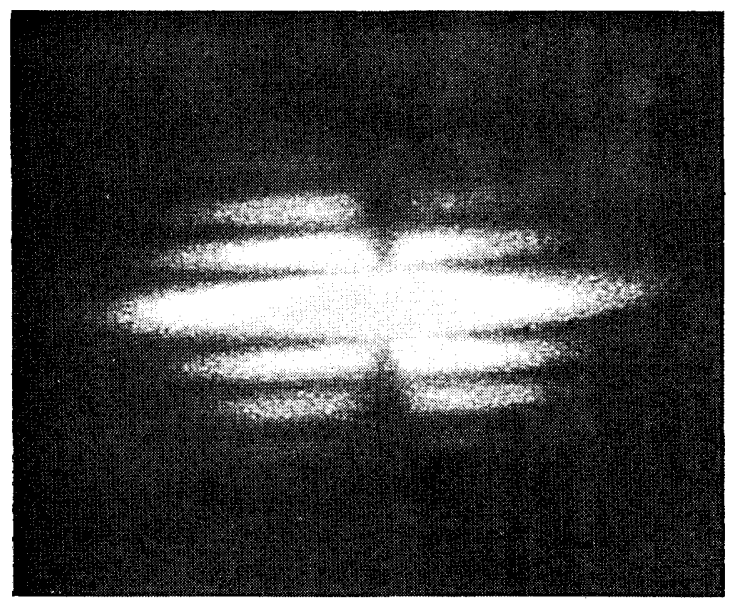

Fig. 4. Quasi-real-time fringes of a vibration measurement of an oscillating tuning fork (amplitude $\rho=13.5 \mu \mathrm{m}$ ). of the pupil of the image forming lens, is modulated by the square of the Bessel function of order zero and of the first kind. The amplitude of vibration is found by substituting for $J_{0}=0$ for $\left(2 \pi x_{1}^{\prime} \rho^{\prime}\right) /\left(\lambda f_{2}^{\prime}\right)=2.40,5.52$, $8.66, \ldots$ Considering the separation of the first minima by $x_{p_{1}}^{\prime}$ the amplitude of oscillation in the object is found when $M$ is the lateral magnification of $L_{1}$ :

$$
\rho=0.76 \frac{f_{2}^{\prime} \lambda_{2}}{M x_{p_{1}}^{1}} .
$$

The amplitude of oscillation can be found from Eq. (9) by measuring the separation of the first minima of the Bessel function $J_{0}$. Higher-order minima can be considered in order to improve the accuracy of the measurements.

For the analysis of torsional oscillations, the timeaverage recording of the speckle pattern occurs in the Fourier transform plane of $L_{1}$ in Fig. $1,{ }^{4}$ for example. It can also be found by defocused speckle pattern recording. ${ }^{7}$

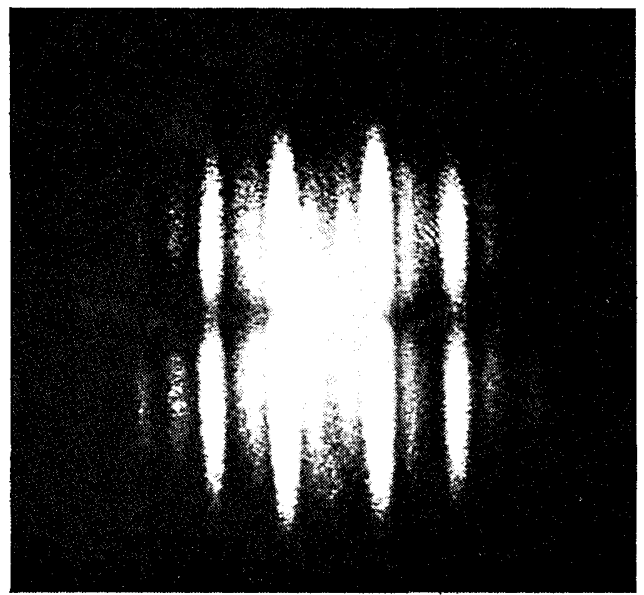

Fig. 5. Horizontally vibrating tuning fork was also shifted horizontally between two exposures $(\Delta \eta=\rho=15 \mu \mathrm{m})$.

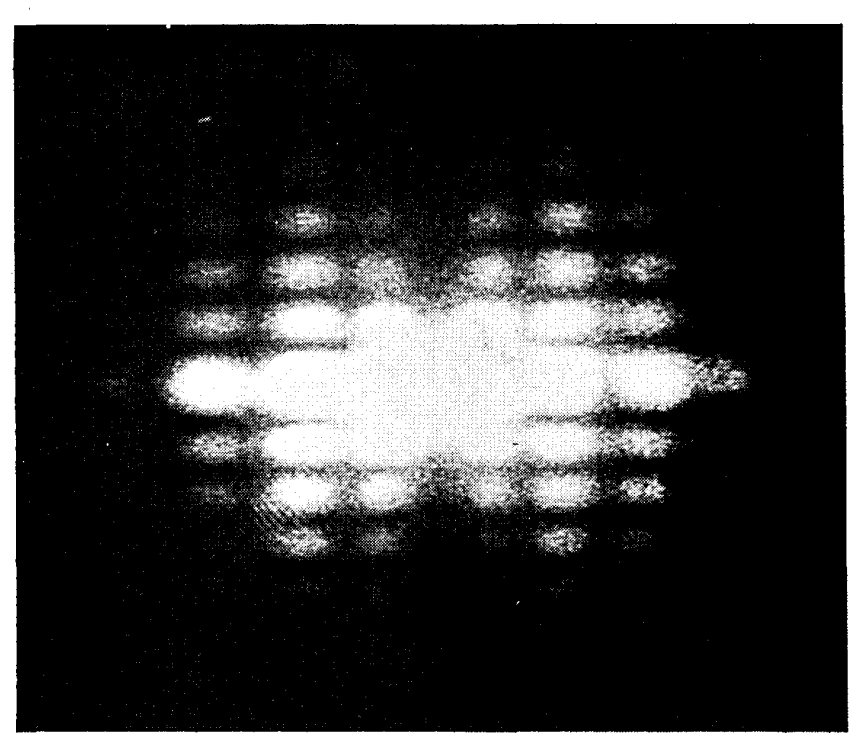

Fig. 6. Horizontally vibrating tuning fork shifted vertically between two exposures $(\Delta \eta=\rho=15 \mu \mathrm{m})$. 


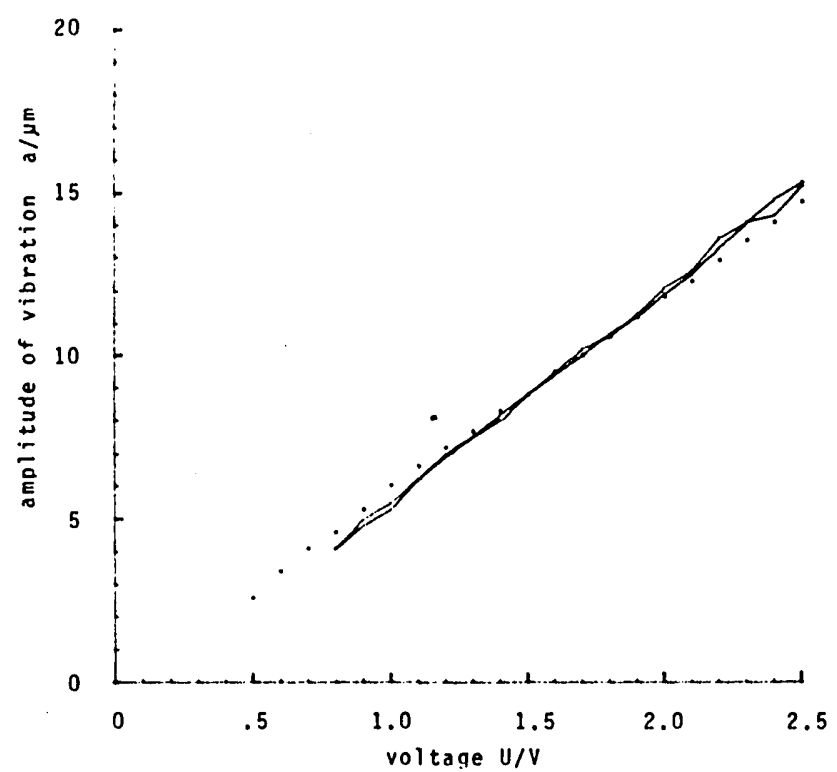

Fig. 7. Comparison of vibration measurements with the speckle technique (solid line, two independent measurements) and a laser Doppler vibrometer (dotted line).

\section{Experimental Results}

A typical arrangement for deformation, displacement, and vibration analysis in quasi-real time using speckle pattern recording is shown in Fig. 1. The object is an optically rough surface illuminated with coherent light from an Ar laser, for example, with the wavelength $\lambda_{1}$ $=514 \mathrm{~nm}$. For in-plane vibration analysis the lens $L_{1}$ forms the image of the oscillating object into the crystal. The power to write the speckle pattern into the BSO crystal was of the order of $20 \mu \mathrm{W} \mathrm{cm}{ }^{-2}$, and the cycling time was $1 \mathrm{sec}$. The field $E_{0}$ applied across the crystal was $5 \mathrm{kV}$, and the crystal size used for the experimental results shown was $10 \times 10 \times 1.5 \mathrm{~mm}^{3}$.

For displaying Young's fringes the BSO was illuminated with a spherical wave of a He-Ne laser with $\lambda_{2}=$ $633 \mathrm{~nm}$ and 1-mW power as indicated in Fig. 1. To avoid stray light, a polarizer and analyzer were used. To eliminate the undiffracted light, a stop can be placed in the center of the Fraunhofer plane. The fringes are displayed on a TV screen. For the analysis of torsional oscillations a similar experimental arrangement is used where the BSO is placed into the Fourier transform plane of the lens $L_{1}$ in Fig. 1.

Real-time fringes of double-exposed speckle patterns with a displacement of $40 \mu \mathrm{m}$ between the exposures are shown in Fig. 2 and with tilts of 20 sec of arc in Fig. 3. The fringe analysis can be carried out using TV techniques as indicated in Fig. 3. Figure 4 shows fringes obtained by time-average recording in the BSO of an oscillating tuning fork oscillating at a frequency of 1 $\mathrm{kHz}$. The fringe patterns of superimposed movements, displacement, and mechanical oscillation are shown in Figs. 5 and 6. In Fig. 5 the time-average speckle pattern of an oscillating tuning fork was shifted horizontally by an object displacement of $15 \mu \mathrm{m}$ in the horizontal direction. The amplitude of oscillation in the same direction was $15 \mu \mathrm{m}$. In Fig. 6 an object shift of $15 \mu \mathrm{m}$ was introduced in the horizontal direction in addition to the oscillation of amplitude of $15 \mu \mathrm{m}$ in the vertical direction. The fringe spacings of the two fringe systems are different as expected from the theory. In Fig. 7 a comparison is shown of the results obtained by the analysis of an oscillating tuning fork with the described real-time novel speckle technique and the vibrometer based on the Doppler shift technique. Good agreement could be obtained between the results of the vibration analysis obtained with two different techniques for different applied voltages.

\section{Conclusions}

Speckle photography applied in real time for deformation, displacement, and vibration analysis was found to be very attractive. The BSO storage material proved to be very promising with unlimited recycling. No storage device is required when the writing and reading out wavelengths are chosen appropriately. The TV techniques with respect to spectral sensitivity of the crystal can be used directly for fringe analysis.

We would like to thank $\mathrm{R}$. Litschel for providing the results of the Doppler shift measurements and the DFG for financial support.

\section{References}

1. J. P. Huignard and F. Micheron, Appl. Phys. Lett. 29, 591 (1976).

2. J. P. Huignard and J. P. Herriau, Appl. Opt. 16, 1807 (1977).

3. J. P. Huignard, J. P. Herriau, G. Rivet, and P. Günter, Opt. Lett. 5, 102 (1980).

4. H. J. Tiziani, K. Leonhardt, and J. Klenk, Opt. Commun. 34, 327 (1980).

5. T. Takemori, S. Ueha, and J. Tsujiuchi, Opt. Commun. 32, 24 (1980).

6. H. J. Tiziani, Opt. Acta 18, 891 (1971).

7. D. A. Gregory, Opt. Laser Technol. 8, 201 (1976). 